\title{
Síndrome de Sjögren primária: aspectos relevantes para os dentistas
}

\begin{abstract}
- Wanessa Siqueira Cavalcante Departamento de Dermatologia, Faculdade de Medicina, Universidade de São Paulo, São Paulo, SP, Brasil - Silvia Vanessa Lourenço Departamento de Patologia Geral, Faculdade de Odontologia, Universidade de São Paulo, São Paulo, SP, Brasil • Giovanna Piacenza Florezi Departamento de Diagnóstico Bucal, Faculdade de Odontologia, Universidade de São Paulo, São Paulo, SP, Brasil • Sheyla Batista Bologna Departamento de Diagnóstico Bucal, Faculdade de Odontologia, Universidade de São Paulo, São Paulo, SP, Brasil • Ricardo Hsieh Departamento de Diagnóstico Bucal, Faculdade de Odontologia, Universidade de São Paulo, São Paulo, SP, Brasil • Jaqueline Vaz Vanini Departamento de Diagnóstico Bucal, Faculdade de Odontologia, Universidade de São Paulo, São Paulo, SP, Brasil • Milena Monteiro de Souza Antunes Departamento de Dermatologia, Faculdade de Medicina, Universidade de São Paulo, São Paulo, SP, Brasil • Fernanda de Paula Departamento de Diagnóstico Bucal, Faculdade de Odontologia, Universidade de São Paulo, São Paulo, SP, Brasil • Sandra Gofinet Pasoto Departamento de Reumatologia, Faculdade de Medicina, Universidade de São Paulo, São Paulo, SP, Brasil • Luiz Fernando Ferraz da Silva Departamento de Patologia, Faculdade de Medicina, Universidade de São Paulo, São Paulo, SP, Brasil • Marcello Menta Simonsen Nico Departamento de Dermatologia, Faculdade de Medicina, Universidade de São Paulo, São Paulo, SP, Brasil
\end{abstract}

RESUMO | Objetivo: Esta revisão de literatura tem o propósito de oferecer aos cirurgiões-dentistas entendimento sobre os principais achados clínico-epidemiológicos da síndrome de Sjögren primária (SSp), bem como ressaltar os principais aspectos etiopatogênicos e histopatológicos da doença. Métodos: Buscou-se na base de dados Pubmed/Medline e ScienceDirect os descritores "síndrome de Sjögren", "glândulas salivares", "xerostomia", "diagnóstico", "glândula salivar menor", "etiologia", "biopsia” entre os anos de 1991 a 2017. Discussão: A SSp é uma exocrinopatia autoimune que tem como substrato, em especial, as glândulas lacrimais e salivares, culminando com os sintomas de xeroftalmia e xerostomia. Seu principal achado morfológico é a presença de agregados de leucócitos mononucleares ao redor dos ductos e ácinos. Outras manifestações sistêmicas podem estar presentes, acarretando considerável morbidade nos pacientes acometidos. Atualmente, foi publicado o mais novo conjunto de critérios diagnósticos da doença, sendo um marco histórico no diagnóstico precoce da enfermidade, fundamental para diminuir os altos números de subdiagnósticos e otimizar a conduta clínica. Conclusão: Esta revisão abordou os aspectos clínicos-epidemiológicos, histopatológicos e etiopatogênicos da SSp, objetivando melhor compreensão dessa doença multifatorial e, portanto, a necessidade de uma conduta multidisciplinar e do papel fundamental do cirurgião-dentista no manejo dos pacientes acometidos por essa síndrome.

\section{DESCRITORES | Síndrome de Sjögren; Glândulas Salivares; Xerostomia; Patogênese.}

ABSTRACT | Primary Sjögren's syndrome: relevant aspects to dentists - Objective: The aim of this present review is to provide dentists a better understanding of Primary Sjögren's syndrome (SSp), clinical and epidemiological findings, also the etiopathogenic and histopathological features of the disease. Methods: The search was based on Pubmed/Medline and ScienceDirect database from 1991 to 2017. It was used keywords: "Sjögren’s syndrome", "salivary gland", "xerostomia", "pathogenesis", "diagnosis", "minor salivary gland", "ethiology”, "biopsy”. Discussion: Primary Sjögren's syndrome (SSp) is an autoimmune exocrinopathy involving mainly the lacrimal and salivary glands, resulting in reduced secretory functions and classical sicca symptoms, such as dry eyes and dry mouth. The hallmark of the histopathological features is the presence of aggregates of mononuclear leukocytes surrounding ducts and acini. Others systemic manifestations can also be present, leading to considerable morbidity in the affected patients. The lastest diagnosis criteria of SSp was published in 2017, becoming a historical landmark in the early diagnosis of the disease, reducing the high number of underdiagnosis and improving therapeutic intervention. Conclusion: This review approached the clinical-epidemiological and histopathological features, and etiopathogenic factors of SSp, supporting a better comprehension of this multifactorial disease and needing a multidisciplinary approach and the role of the dentist in the clinical conduct of the patients affected by this syndrome.

DESCRIPTORS | Sjögren’s Syndrome; Salivary Glands; Xerostomia; Pathogenesis.

AUTOR CORRESPONDENTE

- Wanessa Siqueira Cavalcante Departamento de Dermatologia, Faculdade de Medicina, Universidade de São Paulo • Av. Doutor Eneas Carvalho de Aguiar, 470, Prédio II, 2 andar São Paulo, SP, Brasil • 05403-000 E-mail: wanessasiqueira@usp.br

- Received Sep 12, 2017 • Accepted Nov 23, 2017

- Dol http://dx.doi.org/10.11606/issn.2357-8041.clrd.2017.137879 


\section{INTRODUÇÃO}

Em 1933, o oftalmologista dinamarquês Henrik Sjögren descreveu os achados clínicos e histológicos da síndrome sicca, caracterizada pela extrema secura da mucosa oral e dos olhos em pacientes do sexo feminino, além de provável artrite reumatoide ${ }^{1}$. Desde então, as pesquisas têm revelado uma importante doença com aspectos clínicos, sorológicos e etiopatogênicos intrigantes².

A síndrome de Sjögren primária (SSp), também denominada epitelite autoimune, é uma alteração autoimune com características presentes em um amplo espectro clínico que se apresenta desde uma exocrinopatia local, com acometimento das glândulas salivares e lacrimais, às múltiplas manifestações sistêmicas ${ }^{3,4}$. Tais manifestações são ainda de difícil avaliação objetiva, ocasionando alto grau de subdiagnóstico ${ }^{2,5}$.

Inicialmente, os principais sintomas são xerostomia e xeroftalmia, frequentemente acompanhadas de alteração sistêmica que afeta órgãos parenquimatosos, como pulmões, rins e fígado, além da associação com eventos linfoproliferativos que podem culminar no desenvolvimento de linfoma ${ }^{6,7}$.

Nessa perspectiva, a diminuição ou perda da secreção salivar é causada pela destruição do parênquima glandular, principalmente em decorrência da inflamação crônica que arruína a estrutura das glândulas salivares ${ }^{8}$. Portanto, o entendimento da etiopatogenia e dos mecanismos de interação da inflamação com as glândulas salivares facilitará a compreensão das manifestações orais, favorecendo a conduta clínica dos cirurgiões-dentistas e, assim, contribuindo para melhor qualidade de vida dos pacientes acometidos.

A busca por artigos foi realizada de forma não sistemática por meio de pesquisa nas bases de dados Pubmed/Medline e ScienceDirect, alternando os descritores "síndrome de Sjögren", "glândulas salivares", "xerostomia”, "diagnóstico", "glândula salivar menor", "etiologia”, "biopsia”, entre 1991 e 2017. Foram selecionados 47 artigos por meio da leitura dos respectivos títulos e resumos. Os artigos que não estavam disponíveis na íntegra foram solicitados à biblioteca da Faculdade de Medicina da Universidade de São Paulo (FMUSP). Posteriormente, todos os manuscritos foram lidos e interpretados criticamente.

\section{DISCUSSÃO}

Tradicionalmente, a SSp é uma doença autoimune bastante estudada por reumatologistas. Em 2002, foi publicado o primeiro consenso internacional sobre os critérios de classificação dessa enfermidade a partir da preposição do The American-European Consensus Group (AECG) ${ }^{9}$.

Em 2012, o American College of Rheumatology (ACR) endossou os novos critérios para a classificação da doença propostos pelo grupo Sjögren's International Collaborative Clinical Alliance (SICCA), os quais foram concentrados em três alterações centrais: ceratoconjuntivite sicca, sialadenite e fatores sorológicos ${ }^{10}$.

No entanto, em 2017, o ACR e a European League Against Rheumatism (Eular) publicaram o mais novo consenso metodológico que foi um "divisor de águas" para o diagnóstico precoce da SSp, assim, pacientes com sinais e sintomas sugestivos da doença podem ser precocemente diagnosticados com base nos critérios objetivos, tais como: exame sorológico positivo, sialadenite focal linfocítica (escore focal $\geq 1$ focos $/ 4 \mathrm{~mm}^{2}$ ), critério objetivo oral e critérios objetivos oculares, conforme descritos no Quadro $1^{11}$.

Essa atual classificação demonstrou possuir alta sensibilidade (96\%) e especificidade (95\%) quando comparada às classificações anteriores, o que a torna um método bem aceito para o diagnóstico assertivo da $\mathrm{SSp}^{11}$. Entretanto, ela não exime a experiência clínica do avaliador diante dos diversos casos. 
ini JV • Antune MIMS • Paula F •

QUADRO 1 | Critérios de classificação da SSp de acordo com o ACR e a Eular ${ }^{10}$.

\begin{tabular}{c|c}
$\begin{array}{c}\text { A classificação de SSp aplica-se a qualquer indivíduo que atenda } \\
\text { aos critérios de inclusãoA } \\
\text { listadas como critérios de exclusão }{ }^{\mathrm{B}} \text { e tenha escore } \geq 4 \\
\text { quando os pesos dos cinco itens são somados: }\end{array}$ \\
\hline $\begin{array}{c}\text { Item } \\
\text { Peso/escore }\end{array}$ \\
$\begin{array}{c}\text { Glândula salivar labial com sialadenite linfocítica } \\
\text { focal e escore focal } \geq 1 \text { focos/4 } \mathrm{mm}^{2} \text { c }\end{array}$ & 3 \\
\hline Anti-SSA/Ro-positivo & 3 \\
\hline $\begin{array}{c}\text { Escore coloração ocular } \geq 5 \text { (ou escore de } \\
\text { van Bijsterveld } \geq 4 \text { ) em pelo menos um olho }\end{array}$ & 1 \\
\hline $\begin{array}{r}\text { Teste de Schirmer } \leq 5 \mathrm{~mm} / 5 \mathrm{~min} . \\
\text { em pelo menos um olho }\end{array}$ & 1 \\
\hline $\begin{array}{c}\text { Taxa do fluxo salivar total } \\
\text { não estimulado } \leq 0,1 \mathrm{ml} / \mathrm{min}\end{array}$ & 1 \\
\hline
\end{tabular}

A Os critérios de inclusão são aplicados a qualquer paciente com pelo menos um sintoma de secura ocular ou oral, definida por resposta positiva a pelo menos uma destas questões: (1) Você teve sensação de olhos secos diariamente e de forma persistente por mais de três meses? (2) Você tem sensação recorrente de areia nos olhos? (3) Você usa lágrima artificial mais de três vezes por dia? (4) Você teve diariamente uma sensação de boca seca por mais de três meses? (5) Você bebe frequentemente líquidos para ajudar na deglutição de alimentos secos?

${ }^{\text {B }}$ Os critérios de exclusão incluem o diagnóstico prévio de qualquer uma das seguintes condições, o que descartaria o diagnóstico de SSp e a participação nos estudos dessa doença ou em ensaios clínicos terapêuticos por causa de características clínicas que se sobrepõem ou de interferência nos testes dos critérios: (1) história de radioterapia na cabeça e no pescoço; (2) infecção por hepatite C ativa (confirmada por reação em cadeira da polimerase - PCR); (3) síndrome da imunodeficiência human - SIDA; (4) sarcoisose; (5) amiloidose; (6) doença enxerto versus hospedeiro; (7) doença relacionada ao lgG4.

${ }^{\mathrm{C}} \mathrm{O}$ exame histopatológico deve ser realizado por um patologista experiente no diagnóstico de sialadenite linfocítica focal e em contagem de focos, usando o protocolo descrito por Daniels et al. ${ }^{12}$.

${ }^{D}$ Pacientes que habitualmente usam medicamentos anticolinérgicos devem ser avaliados quanto aos sinais objetivos de hipofunção salivar e secura ocular após o intervalo de tempo suficiente sem essas medicações para que esses componentes sejam uma medida válida da secura oral e ocular.

\section{Aspectos clínicos-epidemiológicos}

De acordo com os critérios estabelecidos ${ }^{9,10}$, a prevalência dessa síndrome na população geral é estimada entre $0,1 \%$ e $0,5 \%{ }^{13,14}$. Embora SSp possa acometer pessoas de todas as idades, há maior incidência nos indivíduos entre a $4^{\mathrm{a}}$ e a $5^{\mathrm{a}}$ décadas de vida, sendo as mulheres mais acometidas do que os homens, numa proporção de 9:1 $1^{15-18}$.

Clinicamente, a secura das mucosas é a característica mais comum encontrada nos pacientes com SSp, principalmente das mucosas oral e ocular. Não obstante, os pacientes podem ter dificuldade para determinar com exatidão o início da doença e, por isso, os sintomas podem ser facilmente ignorados ou mal interpretados ${ }^{19}$, provavelmente devido à baixa especificidade dos sinais cardinais da doença, como representados na Figura $1^{20}$.

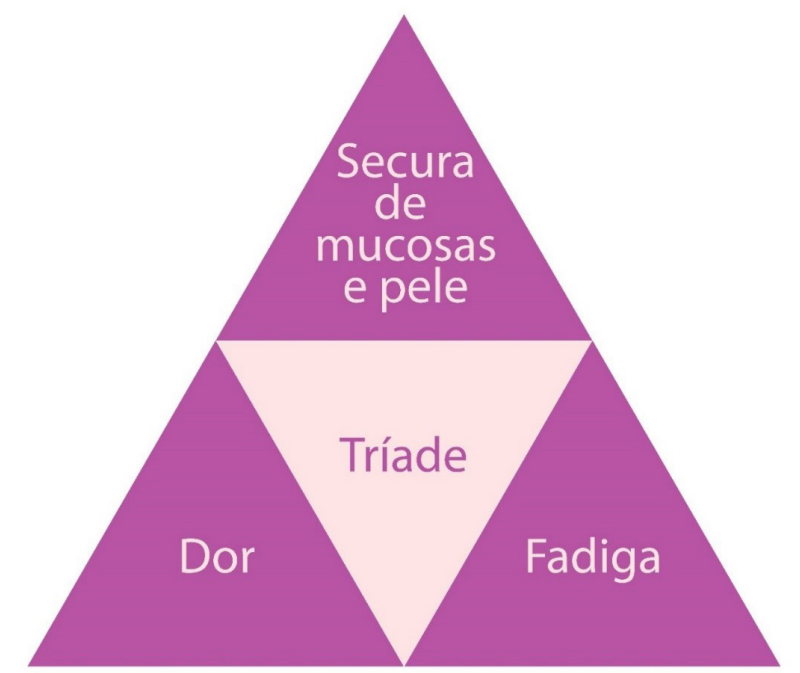

FIGURA 1 | Sinais cardinais da síndrome de Sjögren primária (SSp).

Nessa síndrome, o sintoma de olho seco pode estar presente mesmo quando a aparência clínica dos olhos pareça inalterada. A xeroftalmia é definida pela diminuição da produção de lágrima, capaz de causar destruição da conjuntiva e da bulbar da córnea, quadro clínico denominado ceratoconjuntivite sicca. Os sintomas mais comuns são: ardência, sensação de corpo estranho nos olhos, coceira, vermelhidão e fotossensibilidade ${ }^{21}$. Há efeitos inflamatórios severos que podem culminar na perda da visão, tais como: esclerite, nódulos inflamatórios na superfície e úlcera corneanas ${ }^{19}$.

A SSp é a doença índice relacionada com a hipossalivação e a xerostomia. A primeira é um achado objetivo da diminuição da produção do fluxo salivar e acontece quando a quantidade de saliva produzida for menor ou igual a $0,1 \mathrm{~mL} / \mathrm{min}$; enquanto a outra é definida pela sensação subjetiva de boca seca. O cirurgião-dentista é o profissional 
capacitado para verificação da presença desses critérios, uma vez que possuem importante valor para a elaboração diagnóstica da $\mathrm{SSp}^{22,23}$.

Nesse grupo de pacientes, a redução do fluxo salivar pode ser considerada como um biomarcador da hipofunção das glândulas salivares ${ }^{8,24,25}$, favorecendo o acúmulo de placa dental e o surgimento de cáries dentárias, doenças periodontais e infecções oportunistas $^{2,21,24}$. Nessa doença, a diminuição ou perda da secreção salivar é causada pela destruição do parênquima glandular, principalmente em decorrência da inflamação crônica que destrói a estrutura das glândulas salivares ${ }^{8}$.

Os sinais clínicos frequentemente associados são: perda do brilho, secura, aparência pálida e fina da mucosa oral (Figura 2A), fissuras e lobulações no dorso lingual, queilite angular e atrofia das papilas filiformes. Estes culminam em sintomas como glossodinia (ardência bucal), disgeusia (distorção ou diminuição do paladar), dificuldades na fala, deglutição do bolo alimentar, tosse seca e dificuldade na fixação de próteses dentárias.

Outro sintoma que pode se manifestar é o edema das glândulas salivares maiores, mais comumente das glândulas parótidas, sendo fundamental verificar a existência de infecção bacteriana ascendente ou até mesmo de linfoma ${ }^{21}$. Essas manifestações clínicas podem ser facilmente observadas por cirurgiões-dentistas durante a anamnese e o exame clínico. Não obstante, essas observações podem se tornar o primeiro passo para a descoberta dessa enfermidade ${ }^{2,21,22,24}$.

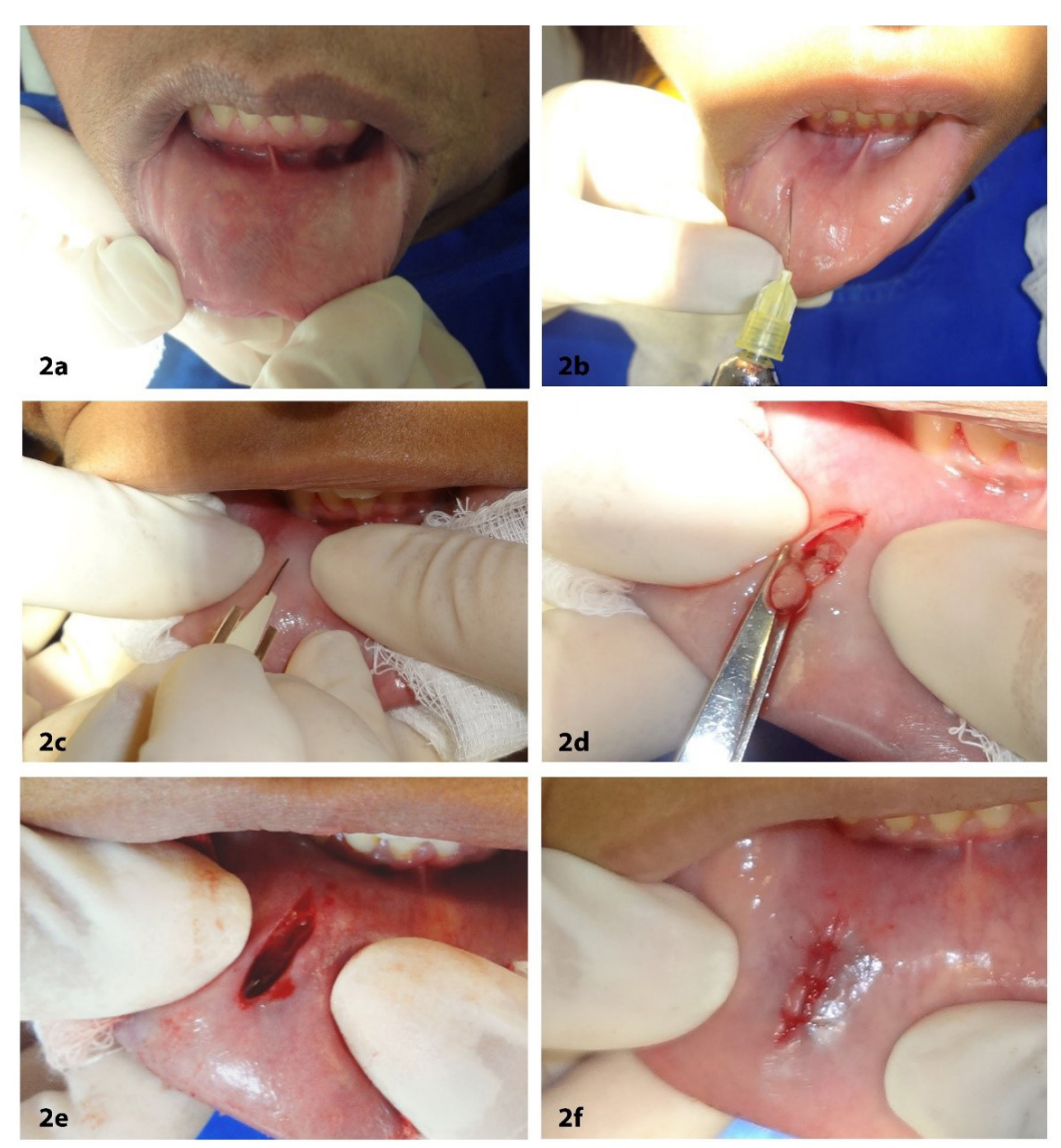

FIGURA 2 | Aspecto da mucosa oral e etapas da biopsia de glândula salivar menor em paciente com SSp. A) Mucosa oral com aspecto ressecado e pálido. B) Anestesia local. C) Incisão. D) Remoção da glândula salivar menor labial pela técnica de retirada individual. E) Remoção de todas as glândulas salivares da área cirúrgica. F) Sutura com coaptação das bordas. 
O diagnóstico diferencial da SSp deve ser realizado com outras enfermidades passíveis de causar xerostomia, tais como: tratamentos radioativos prévios na cabeça e no pescoço; infecção por hepatite C; SIDA; linfoma; sarcoidose; amiloidose; hemocromatose; doença enxerto contra hospedeiro ${ }^{24}$.

Devido à complexidade da doença, os pacientes podem apresentar múltiplas manifestações sistêmicas, como: vasculite; artralgia e artrite; fribromialgia; miopatia; hipomobilidade esofageana; gastrite atrófica; xerotraqueia; doença pulmonar intersticial; bronquiolite; pericardite; neuropatia craniana; polineuropatia periférica; aumento do risco de bloqueio átrio-ventricular total congênito em bebês cujas mães possuam positividade para os autoanticorpos anti-SSA/ Ro e anti-SSB-La ${ }^{3,26}$. Além das manifestações mencionadas, cerca de $5 \%$ a $10 \%$ dos pacientes com SSp podem desenvolver linfoma não Hodgkin, um risco aproximadamente 20 vezes maior do que para a população geral ${ }^{19}$.

\section{Etiopatogenia}

Fatores genéticos, hormonais e ambientais parecem estar relacionados com o desenvolvimento da síndrome, a qual está associada com o aumento da frequência do halotipo HLA-B8/DR $3^{1,4,27}$. Disfunções hormonais podem fazer parte da fisiopatologia de desenvolvimento da SSp, principalmente as deficiências de andrógenos, estrógeno e progesterona $^{28,29}$.

Fatores ambientais e, particularmente, infecções virais atuam como potencial gatilho para o desenvolvimento da $\mathrm{SSp}^{24}$. Vírus como Epstein-Barr (EBV), HTLV-1 (human T leukemia vírus-1), Coxsackievirus e, talvez, o vírus da hepatite $\mathrm{C}$ implicam o desenvolvimento da doença ${ }^{30}$. Entretanto, o principal agente infeccioso envolvido é o vírus $\mathrm{EBV}^{31}$, cuja transmissão ocorre por via oral pelo contato íntimo com a saliva do hospedeiro infectado, resultando em um quadro agudo da mononucleose infecciosa ${ }^{32}$.

Durante a infecção primária, a replicação do vírus EBV ocorre na orofaringe, mais especificamente no interior das células epiteliais da faringe, nas células epiteliais das glândulas salivares e nos linfócitos B. Como consequência dessa infecção, o vírus não é eliminado do organismo, persistindo em sua forma latente para o resto da vida - em pacientes adultos imunocompetentes - ou podendo ser posteriormente reativado ${ }^{33}$.

Na SSp, o material genético do vírus EBV pode ser extraído de amostras teciduais provenientes de material de biopsia de glândulas salivares menores. Postula-se que, assim como outros agentes virais, ele desempenhe papel na patogênese da SSp, atuando como fator desencadeante da doença em indivíduos geneticamente predispostos ${ }^{30}$. De fato, ocorre o mecanismo de mimetismo molecular entre proteínas do vírus EBV e antígenos do hospedeiro, particularmente com o antígeno SSA (Ro), o que pode levar a uma resposta imune humoral dirigida contra os autoantígenos, caracterizando a doença autoimune ${ }^{31}$.

Nesse contexto multifatorial, o modelo de mecanismo de doença na SSp incorpora alterações genéticas, histopatológicas e sorológicas, com repercussões locais e sistêmicas que podem compreender as seguintes etapas (Figura 3):

- agressão inicial ao epitélio glandular (epitelite com alterações arquiteturais) ${ }^{4}$;

- apoptose e apresentação dos autoantígenos anti-SSA (Ro) e anti-SSB (La) na superfície glandular ${ }^{34}$;

- alteração do endotélio intraglandular, produção de proteínas, moléculas de adesão e citocinas pela glândula danificada ${ }^{4}$;

- infiltração e ativação de linfócitos mediada por células apresentadoras de antígenos ${ }^{35}$;

- produção de interferon gama (IFN- $\gamma$ ) pelas células que apresentam antígenos, o que perpetua 
a resposta inflamatória e a secreção de metaloproteinases, as quais irão alterar o parênquima glandular (apoptose) e o estroma ${ }^{36}$.

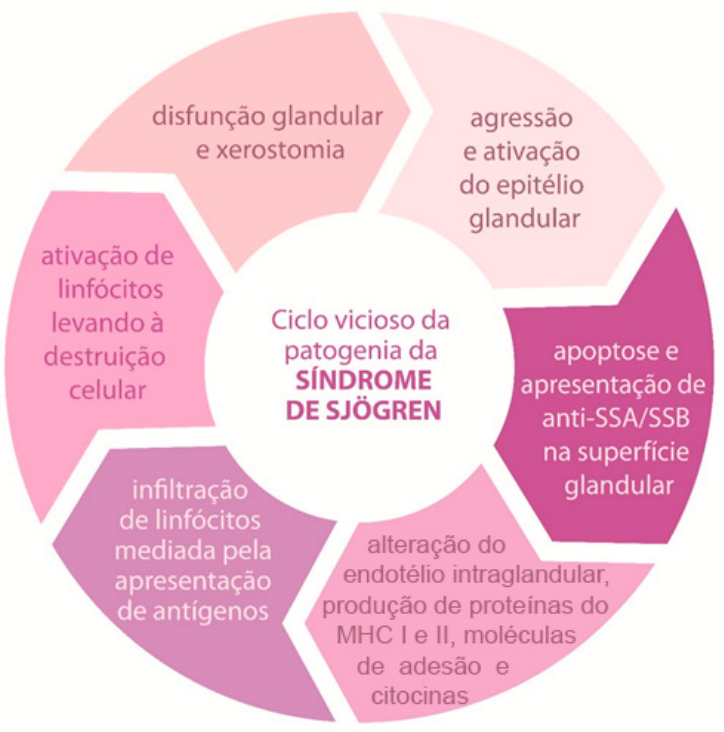

FIGURA 3 | Mecanismo da epitelite autoimune.

Esse complexo mecanismo se transforma então em um ciclo vicioso até a completa destruição do parênquima glandular e, subsequente fibrose, refletindo na qualidade e quantidade de saliva produzida. As alterações morfoarquiteturais resultam em alterações nos canais de aquaporinas, o que altera significativamente a saliva produzida, levando à xerostomia ainda antes da completa destruição da glândula salivar afetada ${ }^{36}$.

\section{Características histopatológicas}

Nos mamíferos, a glândula salivar é estruturalmente composta por ácinos e um sistema de ductos cuja função é conduzir a secreção salivar até a cavidade oral, além de participar ativamente da produção e modificação da saliva, conferindo alta especificidade a esse órgão ${ }^{37}$. Existem três pares de glândulas salivares maiores (parótidas, submandibulares e sublinguais), além de numerosas glândulas salivares menores distribuídas pela mucosa oral ${ }^{25,38-40}$.

O alvo inflamatório na doença é primordialmente o epitélio das glândulas lacrimais e salivares e, por isso, alguns autores ${ }^{4,36}$ cunharam o termo "epitelite autoimune” à destruição do parênquima glandular na SSp, cujos mecanismos iniciais podem estar relacionados à quebra da homeostase na arquitetura glandular - estrutura célula-célula e célula-matriz extracelular ${ }^{4}$.

É consenso a realização de biopsia para diagnóstico da SSp e monitoramento de progressão da doença nas glândulas salivares labiais ${ }^{11}$, pois sua morbidade é baixíssima em relação à biopsia de glândulas salivares maiores, principalmente após o estabelecimento da técnica de retirada individual das glândulas por incisão, conforme representado nas Figuras 2B a 2F. Além disso, essas glândulas são de fácil acesso, estão localizadas sobre uma camada muscular e separadas da mucosa oral por uma fina camada de tecido conjuntivo fibroso. O procedimento cirúrgico é realizado em ambiente ambulatorial por profissionais com conhecimento em estomatologia e precedido por anestesia local ${ }^{41}$.

De acordo com os critérios aceitos pelo $\mathrm{ACR}^{10}$, a característica clássica para o diagnóstico histopatológico da SSp é a presença de sialadenite focal linfocítica, caracterizada por, no mínimo, um foco inflamatório - composto por um agregado de 50 ou mais linfócitos - em $4 \mathrm{~mm}^{2}$ de tecido glandular, em pelo menos 4 lóbulos de glândulas salivares mucosas labiais ${ }^{9}$, o que permite calcular o escore focal e classificá-lo em $<1,1$ ou $\geq 1$ foco inflamatório, conforme proposto por Daniels et al. ${ }^{12}$.

Nesse contexto, a inflamação apresenta-se como o ponto central na destruição do parênquima glandular, diferentemente das glândulas salivares provenientes de indivíduos sem qualquer doença autoimune. Nas glândulas acometidas é possível observar a destruição dos ácinos, extensa fibrose e linfocitose periductal, por análise histopatológica de glândulas salivares menores por profissionais com conhecimento em patologia oral ${ }^{12}$, conforme ilustrado na Figura 4. 

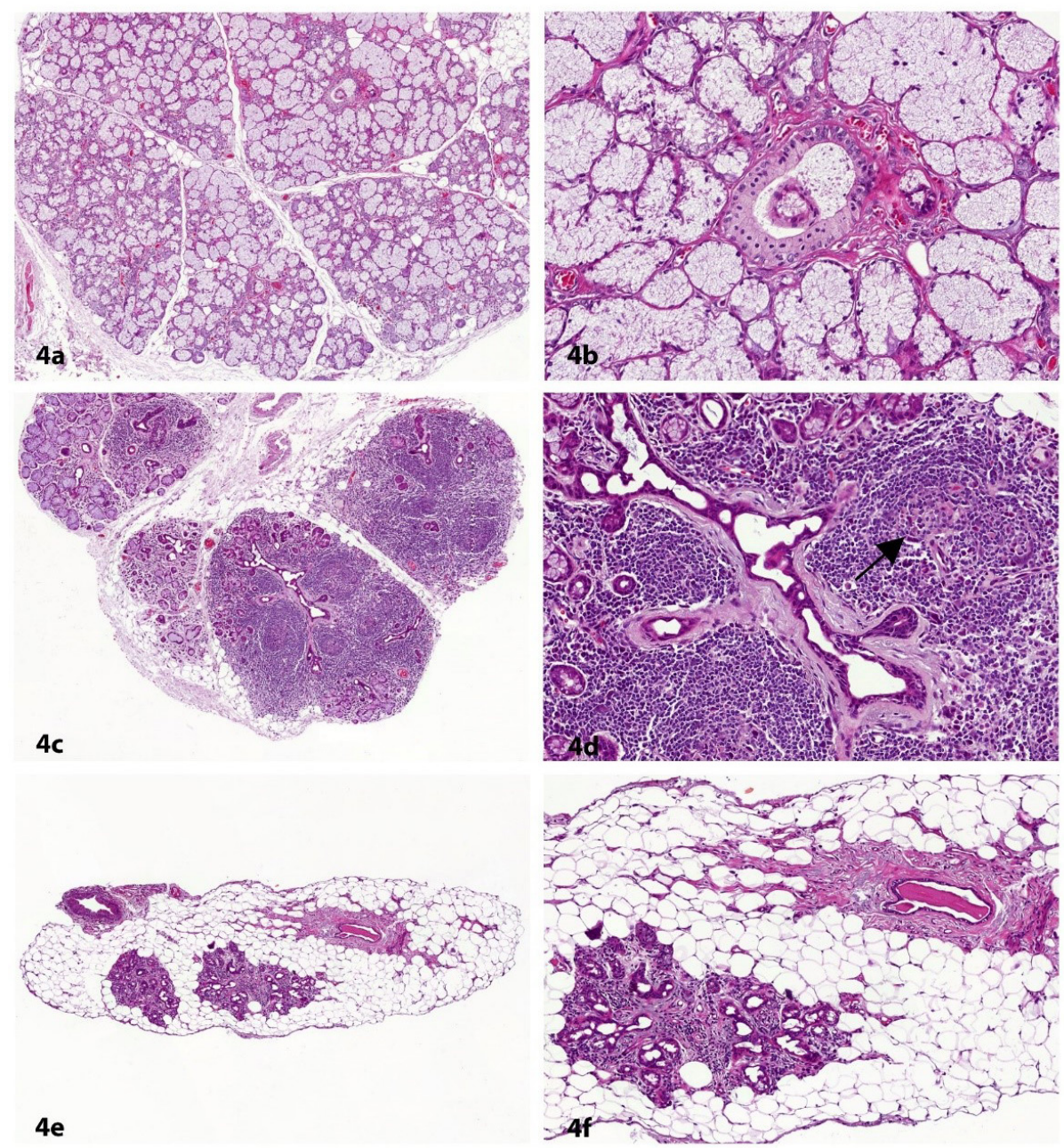

FIGURA 4 Análise histopatológica de glândulas salivares menores. A-B) Glândula salivar labial normal - parênquima glandular composto por lóbulos acinares mucosos, sem infiltrado inflamatório (H\&E, aumento original 50 vezes e 200 vezes, respectivamente). C-D) Glândula salivar labial biopsiada de um paciente diagnosticado com SSp: em C, nota-se grande parte dos lóbulos glandulares substituídos por infiltrado inflamatório intenso, em focos confluentes, áreas de substituição adiposa e fibrose; em D, nota-se, no detalhe, a formação de centros germinativos, que são compostos por agregados linfocíticos densos, dano intenso do parênquima glandular com presença de ilhas epiteliais (seta) e ductos excretores interlobulares com lúmen dilatado (H\&E, aumento original 50 vezes e 200 vezes, respectivamente). E-F) Glândula salivar labial biopsiada de um paciente diagnosticado com SSp: em E, nota-se extensa destruição do parênquima glandular e substituição por tecido adiposo; em F, o detalhe mostra destruição acinar, fibrose e linfocitose periductal (H\&E, aumento original 50 vezes e 200 vezes, respectivamente).

\section{Conduta clínica}

O diagnóstico precoce e o tratamento da SSp são vitais para prevenir as complicações na cavidade oral. A complexidade da síndrome indica que a melhor forma de interferir no problema é por meio da abordagem multidisciplinar, que inclui, no mínimo, a presença de reumatologista, cirurgião-dentista e oftalmologista ${ }^{42,43}$. Infelizmente, a abordagem terapêutica desses pacientes é principalmente sintomática, direcionada para reconhecer e tratar precocemente as complicações da doença ${ }^{21,44}$.

A equipe médica é responsável pela elaboração diagnóstica e pode controlar a sintomatologia sistemicamente a partir de, por exemplo, prescrição 
de imunossupressores e colírios lubrificantes, proporcionando, assim, melhora na qualidade de vida de pacientes com $\mathrm{SSp}^{24}$. No que diz respeito à cavidade oral, as manifestações clínicas são devido à diminuição da secreção salivar e, portanto, o tratamento deve objetivar a minimização dos danos provocados pela xerostomia, o que torna o tratamento odontológico desafiador, pois a falta de saliva reduz a durabilidade das restaurações, aumentando as infiltrações marginais em resinas e promovendo o aumento de lesões cariosas ${ }^{21,44,45}$. De fato, ocorre alteração da microbiota oral nesses pacientes, contribuindo para biofilme com perfil mais cariogênico e acidofílico e elevando, assim, o risco para cáries e candidíase ${ }^{45}$.

Diante desse cenário, as disfunções glandulares podem afetar diretamente a autoestima, as interações sociais e a sensação de bem-estar dos pacientes acometidos ${ }^{46}$. Desse modo, a saúde bucal é um importante pilar no tratamento de pacientes com $\mathrm{SSp}^{45,47}$. Em relação aos cuidados com a saúde bucal, é de suma importância que esses pacientes saibam do risco de desenvolverem lesões cariosas e que estejam envolvidos com os cuidados de sua própria saúde ${ }^{45}$. Desse modo, é fundamental que façam acompanhamento periódico e rigoroso com um cirurgião-dentista, realizem boa higienização bucal utilizando fio dental, façam escovação regular com dentifrício fluoretado, sejam orientados quanto ao padrão de dieta não cariogênica, além do controle de doença periodontal, cáries dentárias e tratamento de infecções oportunistas, como a candidíase, que pode ser resolvida a partir da prescrição de antifúngicos ${ }^{21,24,45,47}$. O Quadro 2 representa um guia prático para o cirurgiãodentista com as principais orientações para o tratamento de manifestações orais em pacientes com SSp ${ }^{45,47}$. Esses pacientes devem, se possível, evitar medicamentos diuréticos, anti-hipertensivos, antidepressivos e anti-histamínicos, pois podem piorar a hipofunção salivar²1.
QUADRO 2 || Guia para o tratamento de manifestações orais em pacientes com SSp ${ }^{45,47}$.

\begin{tabular}{|c|c|}
\hline Manifestação & Recomendação \\
\hline Xerostomia & $\begin{array}{l}\text { Medidas locais para aliviar } \\
\text { sintomas/estimular a salivação: beber } \\
\text { água com frequência; estimulação } \\
\text { mecânica/gustativa: doces sem açúcar, } \\
\text { gomas de mascar, pastilhas. Indicar } \\
\text { utilização de uma saliva artificial: líquido, } \\
\text { gel ou spray; } \\
\text { Evitar alimentos secos, duros, pegajosos e } \\
\text { difíceis de mastigar; } \\
\text { Ingerir líquidos, especialmente água, } \\
\text { durante as refeições. }\end{array}$ \\
\hline $\begin{array}{l}\text { Acometimento } \\
\text { dental }\end{array}$ & $\begin{array}{l}\text { Identificar, trocar ou remover os locais } \\
\text { que proporcionem o acúmulo de placa } \\
\text { (por exemplo, restaurações defeituosas); } \\
\text { Reforçar o compromisso do paciente com } \\
\text { os cuidados sobre a higiene bucal: passar } \\
\text { fio dental e escovar os dentes após as } \\
\text { refeições e, especialmente, antes de dormir; } \\
\text { - Instrução de dieta: reduzir a ingestão de } \\
\text { açúcar, restringir a ingestão de alimentos } \\
\text { açucarados entre as refeições e/ou bebidas } \\
\text { contendo açúcar; } \\
\text { Terapia com flúor em consultório } \\
\text { odontológico: aplicação de flúor gel ou } \\
\text { verniz a cada } 3 \text { meses; } \\
\text { Terapia diária de flúor: dentifrício fluoretado; } \\
\text { Colutório bucal contendo clorexidina (0,12\%) } \\
\text { pode ser indicado para pacientes com alto } \\
\text { risco de desenvolver cárie - bochechar } \\
10 \text { mL do produto, duas vezes ao dia; } \\
\text { Acompanhar o paciente periodicamente é } \\
\text { essencial (pelo menos a cada } 3 \text { meses) e } \\
\text { realizar radiografias interproximais (uma vez } \\
\text { ao ano) para diagnóstico precoce de cárie. }\end{array}$ \\
\hline Candidíase & $\begin{array}{l}\text { Eliminar fatores locais relevantes, } \\
\text { principalmente em pacientes com higiene } \\
\text { oral precária; } \\
\text { Dentaduras ou próteses devem ser } \\
\text { desinfectadas regularmente com clorexidina } \\
(0,12 \%) \text { ou hipoclorito de sódio (1\%) por } \\
30 \text { minutos e seu uso durante o período de } \\
\text { sono noturno deve ser evitado; } \\
\text { Uso intraoral de nistatina, clotrimazol } \\
\text { ou miconazol. }\end{array}$ \\
\hline Outras & $\begin{array}{l}\text { Pomadas, cremes e óleos (a cada } 2 \text { horas) } \\
\text { estão indicados para lábios secos ou com } \\
\text { rachaduras; } \\
\text { Evitar colutórios que contenham álcool, } \\
\text { comidas ácidas ou picantes, e produtos que } \\
\text { contenham tabaco e seus derivados, cafeína } \\
\text { e bebidas alcoólicas; } \\
\text { Usar umidificador de ambiente durante as } \\
\text { horas de sono. }\end{array}$ \\
\hline
\end{tabular}

Adaptado de Gonzáles et al. ${ }^{45} \mathrm{e}$ Cartee et al. ${ }^{47}$. 
A prescrição de saliva artificial, géis lubrificantes ou bochechos pode ser benéfica para pacientes com secura oral severa, no entanto, alguns podem apresentar certa resistência quanto à utilização da saliva artificial devido à curta duração do efeito umectante e ao sabor desagradável ${ }^{21}$. O gel lubrificante Oralbalance ${ }^{\circledR}$ (Laclede Inc, Rancho Dominguez, Califórnia, USA) parece aliviar os sintomas de boca seca e possuir longa duração, sendo adequado também para o uso noturno ${ }^{21,47}$. Outras opções encontradas no mercado brasileiro buscam mimetizar a saliva natural, contendo sais minerais e fluoreto de sódio, cujo objetivo é proporcionar o reequilíbrio da microbiota oral, sensação de conforto e bem-estar.

Por fim, é muito importante que os cirurgiõesdentistas aconselhem seus pacientes quanto à ingestão regular de pequenos goles de água ${ }^{43}$, além de contraindicar o uso de produtos contendo cafeína, cigarros e bebidas alcoólicas ${ }^{44,45}$, atuando, assim, na promoção da saúde ${ }^{21,47}$.

\section{CONCLUSÃO}

Esta revisão abordou os aspectos clínicosepidemiológicos, histopatológicos e etiopatogênicos, levando em consideração os critérios atuais, para o diagnóstico precoce da SSp, e permitindo, desse modo, a melhor compreensão dessa doença multifatorial. É importante ressaltar que uma das principais características dessa enfermidade é a destruição do parênquima das glândulas salivares, com consequente diminuição do fluxo salivar e xerostomia. Diante desse cenário, o cirurgião-dentista exerce papel de extrema importância dentro da equipe multidisciplinar, com instruções meticulosas sobre higiene bucal, aconselhamento de dieta não cariogênica, prescrição de agentes lubrificantes e avaliação constante das restaurações dentais.

Além disso, podem auxiliar os pacientes quanto à escolha de produtos que mimetizem a saliva, prevenir ou remineralizar lesões cariosas incipientes e conduzir o tratamento de infecções oportunistas. Em última análise, os cirurgiões-dentistas são os profissionais capazes de reconhecer precocemente os sinais da xerostomia. A inclusão da SSp como possível diagnóstico diferencial permitirá a comunicação entre os profissionais da saúde sobre a existência desses achados, haja vista que essa colaboração entre os cirurgiões-dentistas e médicos contribuirá para a melhor qualidade de vida dos pacientes com SSp.

\section{REFERÊNCIAS}

1. Fox RI. Sjogren's syndrome. The Lancet. 2005 Jul;366(9482):321-31. doi 10.1016/So140-6736(05)66990-5.

2. Mavragani CP, Moutsopoulos HM. The geoepidemiology of Sjögren's syndrome. Autoimmun Rev. 2010 Mar;9(5):A305-10. doi: 10.1016/j.autrev.2009.11.004.

3. Al-Hashimi I, Khuder S, Haghighat N, Zipp M. Frequency and predictive value of the clinical manifestations in Sjögren's syndrome. J Oral Pathol Med. 2001 Jan;30(1):1-6. doi: 10.1034/ j.1600-0714.2001.300101.x.

4. Mavragani CP, Moutsopoulos HM. Sjögren's syndrome. Annu Rev Pathol. 2014;9:273-85. doi: 10.1146/annurev -pathol-012513-104728.

5. Jonsson R, Vogelsang P, Volchenkov R, Espinosa A, Wahren-Herlenius M, Appel S. The complexity of Sjögren's syndrome: novel aspects on pathogenesis. Immunol Lett. 2011 Dec;141(1):1-9. doi: 10.1016/j.imlet.2011.06.007.

6. Skopouli FN, Dafni U, Ioannidis JP, Moutsopoulos HM. Clinical evolution, and morbidity and mortality of primary Sjögren's syndrome. Semin Arthritis Rheum. 2000 Apr;29(5):296-304. doi: 10.1016/Soo49-0172(00)80016-5.

7. Tzioufas AG, Voulgarelis M. Update on Sjögren's syndrome autoimmune epithelitis: from classification to increased neoplasias. Best Pract Res Clin Rheumatol. 2007 Dec;21(6): 989-1010. doi: 10.1016/j.berh.2007.09.001.

8. Proctor GB. The physiology of salivary secretion. Periodontol 2000. 2016 Feb;70(1):11-25. doi: 10.1111/prd.12116.

9. VitaliC, BombardieriS, Jonsson R, Moutsopoulos HM,Alexander EL, Carsons SE, et al. Classification criteria for Sjögren's syndrome: a revised version of the European criteria proposed by the American-European Consensus Group. Ann Rheum Dis. 2002 Jun;61(6):554-8. doi: 10.1136/ ard.61.6.554. 
10. Shiboski SC, Shiboski CH, Criswell L, Baer A, Challacombe $\mathrm{S}$, Lanfranchi H, et al. American College of Rheumatology classification criteria for Sjögren's syndrome: a data-driven, expert consensus approach in the Sjögren's International Collaborative Clinical Alliance cohort. Arthritis Care Res (Hoboken). 2012 Apr;64(4):475-87.

11. Shiboski CH, Shiboski SC, Seror R, Criswell LA, Labetoulle M, Lietman TM, et al. 2016 American College of Rheumatology/European League against rheumatism classification criteria for primary Sjögren's syndrome: a consensus and data-driven methodology involving three international patient cohorts. Arthritis Rheumatol. 2017 Jan;69(1):35-45. doi: 10.1002/art.39859.

12. Daniels TE, Cox D, Shiboski CH, Schiødt M, Wu A, Lanfranchi $\mathrm{H}$, et al. Associations between salivary gland histopathologic diagnoses and phenotypic features of Sjögren's syndrome among 1,726 registry participants. Arthritis Rheum. 2011 Jul;63(7):2021-30. doi: 10.1002/art.30381.

13. Bowman SJ, Ibrahim GH, Holmes G, Hamburger J, Ainsworth JR. Estimating the prevalence among Caucasian women of primary Sjögren's syndrome in two general practices in Birmingham, UK. Scand J Rheumatol. 2004;33(1):39-43. doi: 10.1080/03009740310004676.

14. Sánchez-Guerrero J, Pérez-Dosal MR, Cárdenas-Velázquez F, Pérez-Reguera A, Celis-Aguilar E, Soto-Rojas AE, et al. Prevalence of Sjögren's syndrome in ambulatory patients according to the American-European Consensus Group criteria. Rheumatology (Oxford). 2005 Feb;44(2):235-40. doi:10.1093/rheumatology/keh455.

15. Bell M, Askari A, Bookman A, Frydrych S, Lamont J, McComb J, et al. Sjögren's syndrome: a critical review of clinical management. J Rheumatol. 1999 Sep;26(9):2051-61.

16. Bowman SJ, Pillemer S, Jonsson R, Asmussen K, Vitali C, Manthorpe R, et al. Revisiting Sjögren's syndrome in the new millennium: perspectives on assessment and outcome measures: report of a workshop held on 23 March 2000 at Oxford, UK. Rheumatology (Oxford). 2001 Oct;40(10):11808. doi: 10.1093/rheumatology/40.10.1180.

17. García-Carrasco M, Ramos-Casals M, Rosas J, Pallarés L, Calvo-Alen J, Cervera R, et al. Primary Sjögren syndrome: clinical and immunologic disease patterns in a cohort of 400 patients. Medicine (Baltimore). $2002 \mathrm{Jul} ; 81(4): 270-80$. doi: 0.1097/01.md.ooooo24210.15761.9 ${ }^{\mathrm{a}}$.

18. Rozman B, Novljan MP, Hocevar A, Ambrozic A, Zigon P, Kveder T, et al. Epidemiology and diagnostics of primary Sjögren’s syndrome. Reumatizam. 2004;51(2):9-12.
19. Sutcliffe N. Sjögren's syndrome. Medicine (Abingdon). 2006 Nov;34(11):476-79. doi.org/10.1053/j.mpmed.2006. 08.006.

20. Nocturne G, Seror R, Devauchelle-Pensec V, Saraux A, Mariette X, Chiche L. Prevalence of primary Sjögren syndrome: what if Sjögren was right after all? Arthritis Care Res (Hoboken). 2017 Feb. doi: 10.1002/acr.23223.

21. Kassan SS, Moutsopoulos HM. Clinical manifestations and early diagnosis of Sjögren syndrome. Arch Intern Med. 2004 Jun;164(12):1275-84. doi: 10.1001/archinte.164.12.1275.

22. Sreebny LM, Schwartz SS. A reference guide to drugs and dry mouth: 2nd edition. Gerodontology. 1997;14(1):33-47. doi: 10.1111/j.1741-2358.1997.00033.x.

23. Plemons JM, Al-Hashimi I, Marek CL, American Dental Association Council on Scientific Affairs. Managing xerostomia and salivary gland hypofunction: executive summary of a report from the American Dental Association Council on Scientific Affairs. J Am Dent Assoc. 2014 Aug;145(8):867-73. doi 10.14219/jada.2014.44.

24. Bowman SJ. Sjögren syndrome. Medicine (Abingdon). 2010 Feb; 38(2):105-8. doi.org/10.1016/j.mpmed.2009.10.011.

25. Turner MD. Hyposalivation and Xerostomia: Etiology, Complications, and Medical Management. Dent Clin North Am. 2016 Apr;6o(2):435-43. doi: 10.1016/j.cden.2015.11.003.

26. Vissink A, Bootsma H, Kroese FG, Kallenberg CG. How to assess treatment efficacy in Sjögren's syndrome? Curr Opin Rheumatol. 2012 May;24(3):281-9. doi: 10.1097/ BOR.obo13e3283524c37.

27. Ramos-Casals M, Tzioufas AG, Stone JH, Siso A, Bosch X. Treatment of primary Sjögren syndrome: a systematic review. JAMA. 2010 jul;304(4):452-60. doi: 10.1001/jama.2010.1014.

28. Hansen A, Lipsky PE, Dörner T. New concepts in the pathogenesis of Sjögren syndrome: many questions, fewer answers. Curr Opin Rheumatol. 2003 Sep;15(5):563-70.

29. Hayashi Y, Arakaki R, Ishimaru N. Apoptosis and estrogen deficiency in primary Sjögren syndrome. Curr Opin Rheumatol. 2004 Sep;16(5):522-6. doi: 10.1097/01. bor.00o0135450.78047.78.

30. Delaleu N, Jonsson MV, Appel S, Jonsson R. New concepts in the pathogenesis of Sjögren's syndrome. Rheum Dis Clin North Am. 2008;34(4):833-45. doi: 10.1016/j.rdc.2008.08.004.

31. Pasoto SG, Natalino RR, Chakkour HP, Viana VST, Bueno C, Leon EP, et al. EBV reactivation serological profile in primary Sjögren's syndrome: an underlying trigger of active articular involvement? Rheumatol Int. 2013 May;33(5):1149-57. doi: 10.1007/s00296-012-2504-3. 
32. Hislop AD. Early virological and immunological events in Epstein-Barr virus infection. Curr Opin Virol. 2015 Dec;15:75-9. doi: 10.1016/j.coviro.2015.08.002.

33. Mariette X, Gozlan J, Clerc D, Bisson M, Morinet F. Detection of Epstein-Barr virus DNA by in situ hybridization and polymerase chain reaction in salivary gland biopsy specimens from patients with Sjögren's syndrome. Am J Med. 1991 Mar;90(3):286-94. doi: 10.1016/0002-9343(91)80007-9.

34. Yannopoulos DI, Roncin S, Lamour A, Pennec YL, Moutsopoulos HM, Youinou P. Conjunctival epithelial cells from patients with Sjögren's syndrome inappropriately express major histocompatibility complex molecules, La(SSB) antigen, and heat-shock proteins. J Clin Immunol. 1992;12(4):259-65. doi: 10.1007/BFoo918149.

35. Routsias JG, Tzioufas AG. Sjögren's syndrome: study of autoantigens and autoantibodies. Clin Rev Allergy Immunol. 2007 Jun;32(3):238-51. doi: 10.1007/s12016-007-8003-8.

36. Barrera MJ, Bahamondes V, Sepúlveda D, Quest AF, Castro I, Cortés J, et al. Sjögren's syndrome and the epithelial target: a comprehensive review. J Autoimmun. 2013 May;42:7-18. doi: 10.1016/j.jaut.2013.02.001.

37. Tandler B. Introduction to mammalian salivary glands. Microsc Res Tech. 1993 Sep;26(1):1-4. doi: 10.1002/jemt.1070260102. 38. Tucker AS. Salivary gland development. Semin Cell Dev Biol. 2007 Apr;18(2):237-44. doi: 10.1016/j.semcdb.2007.01.006.

39. Holmberg KV, Hoffman MP. Anatomy, biogenesis and regeneration of salivary glands. Monogr Oral Sci. 2014;24:1-13. doi: 10.1159/000358776.
40. Varga G. Physiology of the salivary glands. Surgery. 2015 Dec; 33(12) 581-86. doi: 10.1016/j.mpsur.2015.09.003.

41. Delli K, Vissink A, Spijkervet FKL. Salivary gland biopsy for Sjögren's syndrome. Oral Maxillofac Surg Clin North Am. 2014 Feb;26(1):23-33.doi: 10.1016/j. coms.2013.09.005.

42. Valim V, Trevisani VFM, Pasoto SG, Serrano EV, Ribeiro SLE, Fidelix TSA, et al. Recommendations for the treatment of Sjögren's syndrome. Rev Bras Reumatol. 2015 Sep-Oct;55(5):446-57. doi: 10.1016/j.rbr.2015.07.004.

43. Both T, Dalm VASH, van Hagen PM, van Daele PLA. Reviewing primary Sjögren's syndrome: beyond the dryness: From pathophysiology to diagnosis and treatment. Int J Med Sci. 2017;14(3):191-200. doi: 10.7150/ijms.17718.

44. Stefanski AL, Tomiak C, Pleyer U, Dietrich T, Burmester GR, Dorner T. The Diagnosis and Treatment of Sjögren's syndrome. Dtsch Arztebl Int. 2017 May;114(20):354-61. doi: 10.3238/arztebl.2017.0354.

45. González S, Sung H, Sepúlveda D, González M, Molina C. Oral manifestations and their treatment in Sjögren's syndrome. Oral Dis. 2014 Mar;20(2):153-61. doi: 10.1111/odi.12105.

46. Soto-Rojas AE, Kraus A. The oral side of Sjögren syndrome: diagnosis and treatment: a review. Arch Med Res. 2002 Mar-Apr;33(2):95-106. doi: 10.1016/S0188-4409(01)o0371-X.

47. Cartee DL, Maker S, Dalonges D, Manski MC. Sjögren's syndrome: oral manifestations and treatment, a dental perspective. J Dent Hyg. 2015 Dec;89(6):365-71. 